\title{
A NEW THREE-PARAMETER PARALOGISTIC DISTRIBUTION: ITS PROPERTIES AND APPLICATION
}

\author{
R. Idemudia ${ }^{1}$, N. Ekhosuehi ${ }^{2 *}$ \\ ${ }^{1,2}$ Department of Mathematics, University of Benin, Benin City, Nigeria.
}

\begin{abstract}
In this paper, we considered a new generalization of the paralogistic distribution which we called the three-parameter paralogistic distribution. Some properties of the new distribution which includes the survival function, hazard function, quantile function, moments, Renyi entropy and the maximum likelihood estimation (MLE) of its parameters are obtained. A simulation study shows that the MLE of the parameters of the new distribution is consistent and asymptotically unbiased. An applicability of the new three-parameter paralogistic distribution was subject to a real lifetime data set alongside with some related existing distributions such as the Paralogistic, Gamma, Transformed Beta, Log-logistic and Inverse paralogistic distributions. The results obtained show that the new three-parameter paralogistic distribution was superior to other aforementioned distributions in terms of the Akaike information criterion (AIC) and K-S Statistic values. This claim was further supported by investigating the density plots, P-P plots and Q-Q plots of the distributions for the data set under study.
\end{abstract}

Keywords: Survival function; Hazard function, Quantile function, Moments 


\section{Introduction}

The paralogistic distribution is a sub-model of the generalized beta family which was introduced by McDonald (1984). The probability density function (PDF) and the cumulative distribution function $(\mathrm{CDF})$ of the paralogistic distribution are given respectively by:

$$
\begin{gathered}
f(x)=\frac{\alpha^{2}}{x}\left(1+\left(\frac{x}{c}\right)^{\alpha}\right)^{-(\alpha+1)}\left(\frac{x}{c}\right)^{\alpha} ; x, c, \alpha>0 \\
F(x)=1-\left[1+\left(\frac{x}{c}\right)^{\alpha}\right]^{-\alpha} ; \quad x, c, \alpha>0
\end{gathered}
$$

where $c>0$ is the scale parameter and $\alpha>0$ is the shape parameter. Some applications of the paralogistic distribution are seen in economic model of wealth and income, Kleiber and Kotz (2003). Klugman and Persa (1999) used the inverse paralogistic distribution for semi -parametric bivariate distribution. Other related distributions have also been studied: Nadarajah and kotz (2006) introduced the beta exponential distribution, Silva et al. (2010) introduced the beta modified Weilbull, Mahomoudi (2011) studied the beta-Generalized pareto, Alsawarbeh et al. (2012) studied the beta Cauchy distribution, Ismail and Khalid (2014) used the EM algorithm approach to estimate the 2- and 3- parameter Burr type III distribution using complete and censored data. Guerra et al. (2017) proposed the gamma Burr XII distribution, Maksaei and Aitun (2017) developed the Generalized Weibull-Burr XII distribution and applied it to quality control and technical defect data sets. Makubate et al. (2018) proposed the exponentiated Burr XII Weibull distribution and considered not only shape and scale, but also skewness and tail variation. Hamed et al. (2018) developed a new family of pareto distributions called "the $T$-Pareto $\{Y\}$ family of distributions".

The problem with the paralogistic distribution is that when the failure time does not start from the origin, information about the point at which the failure started is not usually known. This problem can be resolved by introducing a shift parameter which can tell us the time of the first failure of the component.

It is in this direction, we introduced a location parameter to the existing paralogistic distribution to obtain the new three parameter paralogistic distribution (NTPLD).

The rest of this paper is organized as follows: in Section 2, we define the new distribution. In Section 3, we present a discussion on some properties of the NTPLD. The MLE of the NTPLD is discussed in Section 4. A simulation study is carried out to examine the performance and accuracy of the MLE of the parameters in section 5. Section 6 covers the application of real a lifetime data set to illustrate the superiority of the NTPLD over some existing models. Section 7 is on discussion of the results while Section 8 concludes the paper. 


\section{The NTPLD}

We introduced the NTPLD which is characterized by shape, scale and location parameters. The cumulative distribution function is defined by

$$
F(x)=1-\left[1+\left(\frac{x-\theta}{c}\right)^{\alpha}\right]^{-\alpha} ; x, c, \alpha>0
$$

and the corresponding density function is given by

$$
f(x)=\frac{\alpha^{2}}{x-\theta}\left(1+\left(\frac{x-\theta}{c}\right)^{\alpha}\right)^{-(\alpha+1)}\left(\frac{x-\theta}{c}\right)^{\alpha} ; x, c, \alpha>0
$$

where $c>0$ is the scale parameter, $\alpha>0$ is the shape parameter and $\theta>0$ is the location parameter. Now, if $\theta=0$ we have the PDF and CDF of the paralogistic distribution given in equations (1.1) and (1.2) respectively.

The survival function, $S(x)$ and hazard rate function, $h(x)$ corresponding to the NTPLD are given respectively by following:

$$
\begin{gathered}
S(x)=\left[1+\left(\frac{x-\theta}{c}\right)^{\alpha}\right]^{-\alpha} \\
h(x)=\frac{\alpha^{2}}{x-\theta}\left(1+\left(\frac{x-\theta}{c}\right)^{\alpha}\right)^{-1}\left(\frac{x-\theta}{c}\right)^{\alpha}
\end{gathered}
$$

Plots of the density of the NTPLD for some fixed values of $\alpha, \theta$ and $\mathrm{c}$ are displayed in Figure 1. A characteristic of the proposed distribution is that its probability density function can accommodate increasing and unimodal shaped forms, which appears to be dependent on the values of the shape parameter. Plots of the survival function and hazard rate function of the NTPLD for some fixed values $\alpha, \theta$ and $\mathrm{c}$ are given in Figures 2 and 3 respectively. A characteristic of the proposed distribution is that its failure rate can accommodate increasing, decreasing and unimodal shaped forms that appears to be dependent on the values of the shape parameter. 


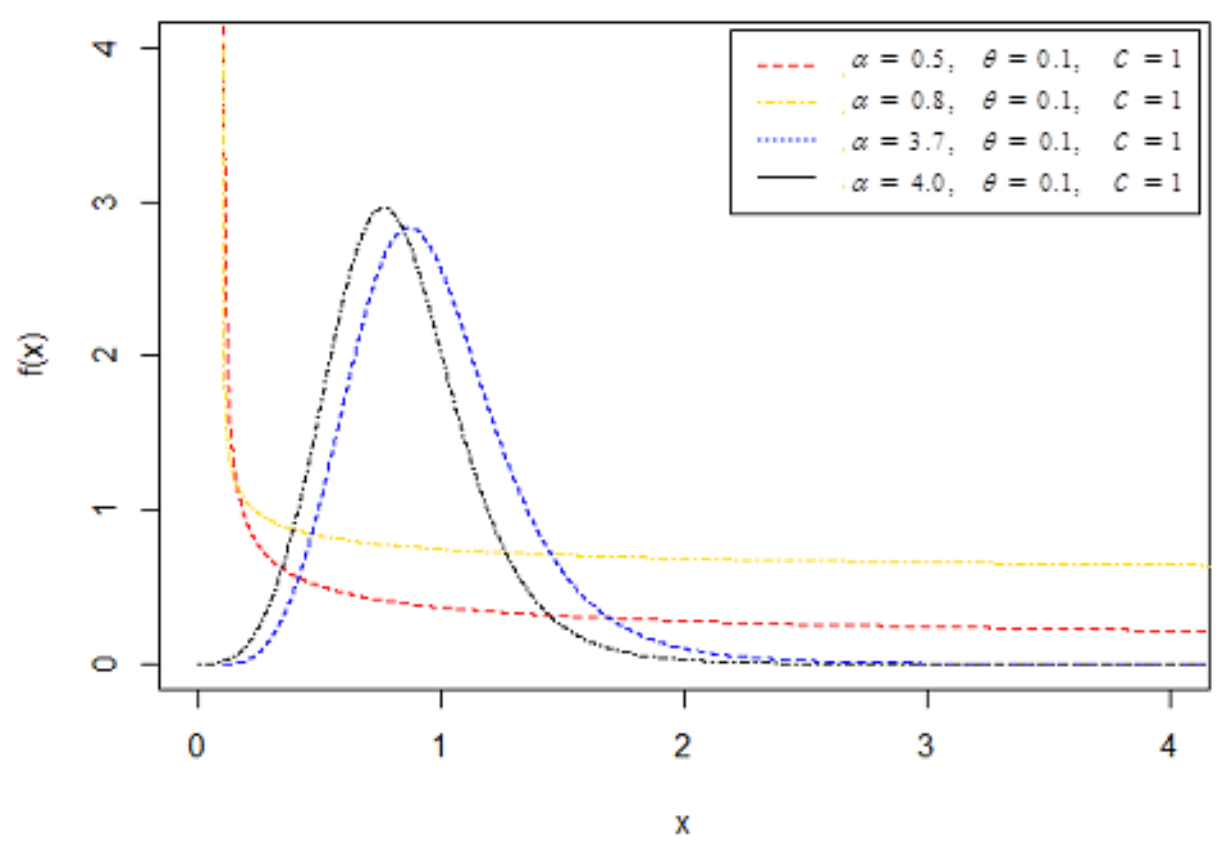

Figure 1. PDF of the NTPLD for fixed values of $(\alpha, \theta, c)=(0.5,0.1,1),(\alpha, \theta, c)=(0.8,0.1,1)$, $(\alpha, \theta, c)=(3.7,0.1,1),(\alpha, \theta, c)=(4,0,1)$

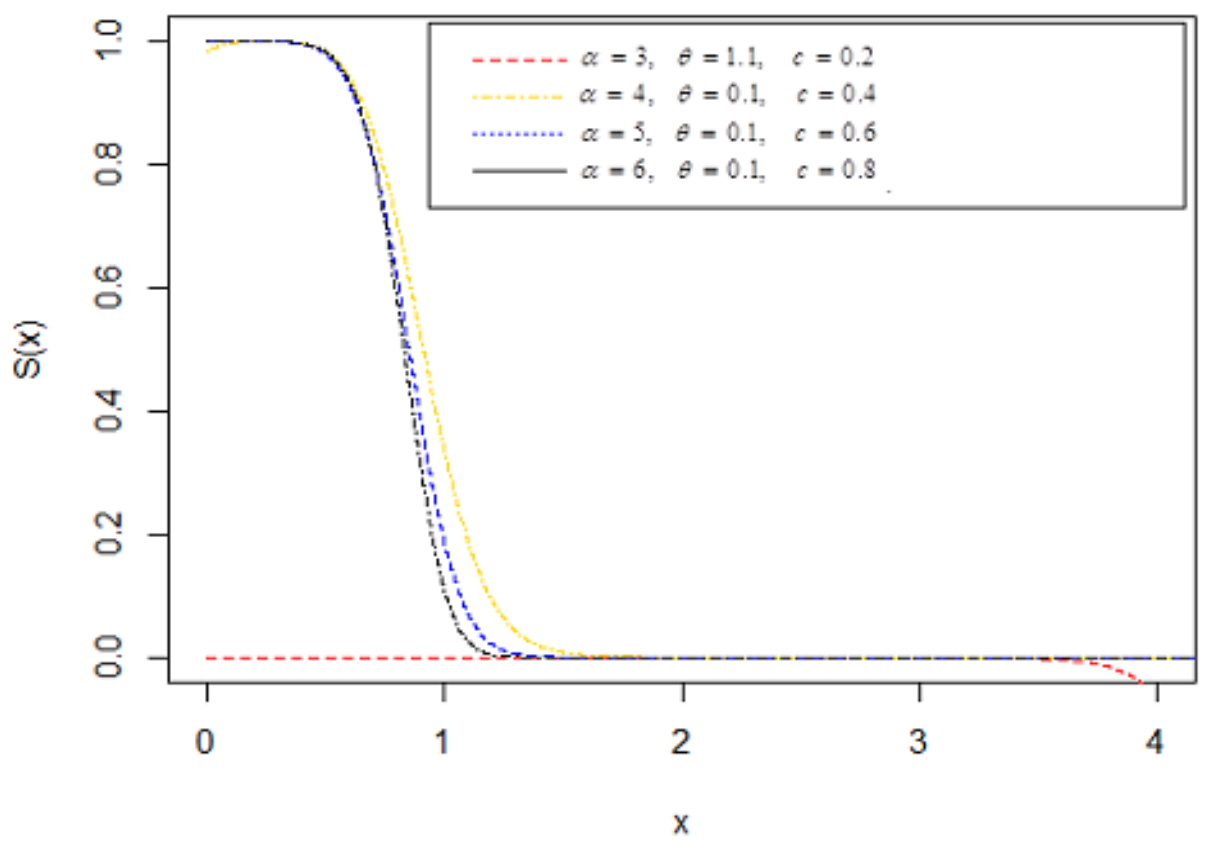

Figure 2: Survival function for some fixed values of $(\alpha, \theta, c)=(3,1.1,0.2),(\alpha, \theta, c)=$ $(4,0.1,0.4),(\alpha, \theta, c)=(5,0.1,0.6),(\alpha, \theta, c)=(6,0.1,0.8)$ 


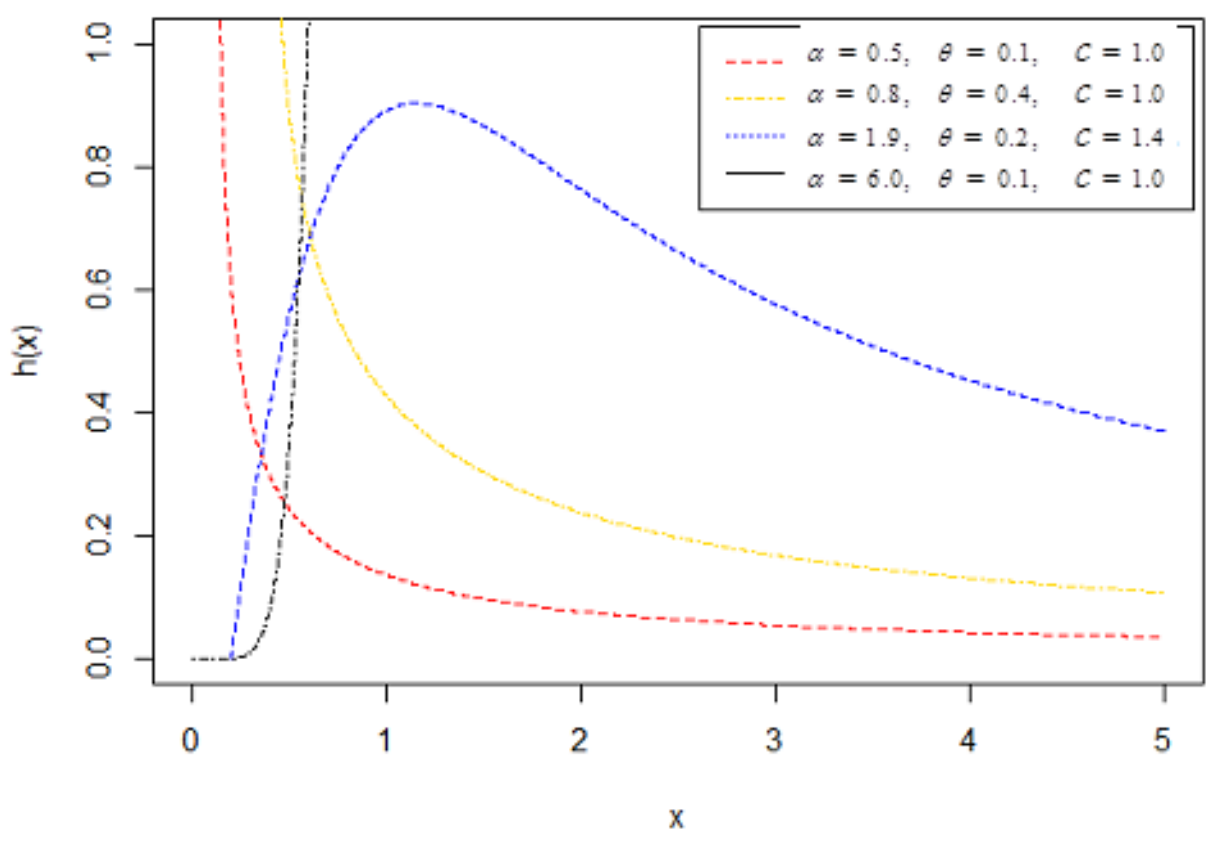

Figure 3: Hazard function of the NTPLD for $(\alpha, \theta, c)=(0.5,0.1,1),(\alpha, \theta, c)=(0.8,0.4,1)$, $(\alpha, \theta, c)=(1.9,0.2,1.4),(\alpha, \theta, c)=(6,0.1,1)$

\section{Some properties of the NTPLD}

\subsection{Moments}

The $r^{\text {th }}$ raw moment of the random variable $X$ is defined by

$$
\mu_{r}^{\prime}=E\left(X^{r}\right)=\int_{\theta}^{\infty} x^{r} f(x) d x
$$

Then, if $X$ follows the NTPLD, we have

$$
\mu_{r}^{\prime}=\int_{\theta}^{\infty} x^{r} \frac{\alpha^{2}}{x-\theta}\left(1+\left(\frac{x-\theta}{c}\right)^{\alpha}\right)^{-(\alpha+1)}\left(\frac{x-\theta}{c}\right)^{\alpha} d x
$$

On further substitution and simplification we have,

$$
\mu_{r}^{\prime}=\alpha \sum_{i=0}^{r}\left(\begin{array}{l}
r \\
i
\end{array}\right) \theta^{r-i} c^{i} \beta\left(\frac{i+\alpha}{\alpha}, \frac{\alpha^{2}-i}{\alpha}\right), r=1,2, \ldots
$$

where $\beta(x, y)=\int_{0}^{\infty} \frac{t^{x-1}}{(1+t)^{x+y}} d t$ is obtained from the relationship between beta and gamma function

Thus, the first four raw moments about the origin of the NTPLD are obtained using equation (3.3):

$$
\mu_{1}^{\prime}=\theta+\frac{c\left(\frac{1}{\alpha}\right) !\left(\frac{\alpha(\alpha-1)-1}{\alpha}\right) !}{(\alpha-1) !}
$$




$$
\begin{gathered}
\mu_{2}^{\prime}=\theta^{2}+\frac{2 \theta c\left(\frac{1}{\alpha}\right) !\left(\frac{\alpha(\alpha-1)-1}{\alpha}\right) !}{(\alpha-1) !}+\frac{c^{2}\left(\frac{2}{\alpha}\right) !\left(\frac{\alpha(\alpha-1)-2}{\alpha}\right) !}{(\alpha-1) !} \\
\mu_{3}^{\prime}=\theta^{3}+\frac{3 \theta^{2} c\left(\frac{1}{\alpha}\right) !\left(\frac{\alpha(\alpha-1)-1}{\alpha}\right) !}{(\alpha-1) !}+\frac{3 \theta^{2} c\left(\frac{2}{\alpha}\right) !\left(\frac{\alpha(\alpha-1)-2}{\alpha}\right) !}{(\alpha-1) !} \\
\mu_{4}^{\prime}=\theta^{4}+\frac{4 \theta^{3} c\left(\frac{1}{\alpha}\right) !\left(\frac{\alpha(\alpha-1)-1}{\alpha}\right) !}{(\alpha-1) !}+\frac{6 \theta^{2} c^{2}\left(\frac{2}{\alpha}\right) !\left(\frac{\alpha(\alpha-1)-2}{\alpha}\right) !\left(\frac{\alpha(\alpha-1)-3}{\alpha}\right) !}{(\alpha-1) !} \\
+\frac{4 \theta c^{3}\left(\frac{3}{\alpha}\right) !\left(\frac{\alpha(\alpha-1)-3}{\alpha}\right) !}{(\alpha-1) !}+\frac{c^{4}\left(\frac{4}{\alpha}\right) !\left(\frac{\alpha(\alpha-1)-4}{\alpha}\right) !}{(\alpha-1) !}
\end{gathered}
$$

Similarly, the $r^{t h}$-Central moment of $X$ is defined by:

$$
\begin{gathered}
\mu_{r}=E\left[(X-\mu)^{r}\right]=\mathrm{E}\left[\sum_{i=0}^{r}\left(\begin{array}{l}
r \\
i
\end{array}\right) X^{r-i}(-\mu)^{i}\right] \\
=\sum_{i=0}^{r}\left(\begin{array}{l}
r \\
i
\end{array}\right) E\left(X^{r-i}\right)(\mu)^{i} \\
=\sum_{i=0}^{r}\left(\begin{array}{l}
r \\
i
\end{array}\right) \mu_{(r-i)}^{i}(-\mu)^{i}
\end{gathered}
$$

where $\mu_{0}=1$ and $\mu_{1}^{\prime}=\mu$

Using equation (3.5), the $2^{\text {nd }}, 3^{\text {rd }}$ and $4^{\text {th }}$ central moments can be obtained using the following:

$$
\begin{gathered}
\mu_{2}=\mu_{2}^{\prime}-\mu^{2} \\
\mu_{3}=\mu_{3}^{\prime}-3 \mu \mu_{2}^{1}+2 \mu^{3} \\
\mu_{4}=\mu_{4}^{\prime}-4 \mu_{3}^{\prime} \mu+6 \mu_{2}^{\prime} \mu^{2}-3 \mu^{4}
\end{gathered}
$$

where the first raw moment and the second central moment corresponds to the mean and variance respectively. 
Thus, the variance $\left(\sigma^{2}\right)$, coefficient of variation $\left(C_{v}\right), \operatorname{skewness}\left(S_{k}\right)$ and $\operatorname{kurtosis}\left(K_{s}\right)$ can be obtained as follows:

$$
\begin{gathered}
\operatorname{Variance}\left(\sigma^{2}\right)=\mu_{2}^{\prime}-\mu^{2} \\
\left(C_{v}\right)=\frac{\sigma}{\mu} \\
\left(S_{k}\right)=\frac{\mu_{3}^{\prime}}{\left(\mu_{2}^{\prime}\right)^{\frac{3}{2}}} \\
\left(K_{s}\right)=\frac{\mu_{4}^{\prime}}{\left(\mu_{2}^{2}\right)}
\end{gathered}
$$

where

$$
\begin{aligned}
& \mu_{3}^{\prime}=\theta^{3}+\frac{3 \theta^{2} c\left(\frac{1}{\alpha}\right) !\left(\frac{\alpha(\alpha-1)-1}{\alpha}\right) !}{(\alpha-1) !}+\frac{3 \theta c^{2}\left(\frac{2}{\alpha}\right) !\left(\frac{\alpha(\alpha-1)-2}{\alpha}\right) !}{(\alpha-1) !} \\
& +\frac{c^{3}\left(\frac{3}{\alpha}\right) !\left(\frac{\alpha(\alpha-1)-3}{\alpha}\right) !}{(\alpha-1) !} \\
& \mu_{4}^{\prime}=\theta^{4}+\frac{4 \theta^{3} c\left(\frac{1}{\alpha}\right) !\left(\frac{\alpha(\alpha-1)-1}{\alpha}\right) !}{(\alpha-1) !}+\frac{6 \theta^{2} c^{2}\left(\frac{2}{\alpha}\right) !\left(\frac{\alpha(\alpha-1)-2}{\alpha}\right) !}{(\alpha-1) !} \\
& +\frac{4 \theta c^{3}\left(\frac{3}{\alpha}\right) !\left(\frac{\alpha(\alpha-1)-3}{\alpha}\right) !}{(\alpha-1) !}+\frac{c^{4}\left(\frac{4}{\alpha}\right) !\left(\frac{\alpha(\alpha-1)-4}{\alpha}\right) !}{(\alpha-1) !}
\end{aligned}
$$

\subsection{Quantile function}

The quantile function of the NTPLD can be obtained by solving for $\mathrm{x}$ in the following equation:

$$
\boldsymbol{\mu}=\boldsymbol{F}(\boldsymbol{x})
$$

where $u$ is a uniform distribution which takes on value between 0 and 1 and $F(x)$ is the CDF of the NTPLD. This quantile function is given by:

$$
Q(u)=\left[(1-\mu)^{\frac{1}{\alpha}}-1\right]^{\frac{1}{\alpha}}
$$

Subsequently, random samples from NTPLD can be generated using equation (3.10) by setting $x=Q(u)$. Table 2 gives the quantiles from the NTPLD for some fixed parameter values. 
Table 1: Moments of the NTPLD for some selected parameter values.

\begin{tabular}{ccccc}
\hline & $(\boldsymbol{\theta}=\mathbf{1 . 1} \boldsymbol{\alpha}=\mathbf{4 . 8}$, & $(\boldsymbol{\theta}=\mathbf{1}, \boldsymbol{\alpha}=\mathbf{4 . 5}$, & $(\boldsymbol{\theta}=\mathbf{1 . 2}, \boldsymbol{\alpha}=\mathbf{4 . 6}$, & $(\boldsymbol{\theta}=\mathbf{1 . 1}, \boldsymbol{\alpha}=\mathbf{4 . 8}$, \\
& $\boldsymbol{c}=\mathbf{0 . 8})$ & $\boldsymbol{c}=\mathbf{0 . 4})$ & $\boldsymbol{c}=\mathbf{0 . 6})$ & $\boldsymbol{c}=\mathbf{0 . 8})$ \\
\hline $\boldsymbol{\mu}_{\mathbf{1}}^{\prime}=\boldsymbol{\mu}$ & 1.5336 & 1.2699 & 1.6056 & 1.2336 \\
$\boldsymbol{\mu}_{\mathbf{2}}^{\prime}$ & 2.3538 & 1.6183 & 2.5900 & 2.6482 \\
$\boldsymbol{\mu}_{\mathbf{3}}^{\prime}$ & 3.6154 & 2.1968 & 4.1977 & 4.5359 \\
$\boldsymbol{\mu}_{\boldsymbol{4}}^{\prime}$ & 5.5573 & 2.6547 & 6.8174 & 7.6190 \\
$\boldsymbol{\sigma}^{\mathbf{2}}$ & 0.0019 & 0.0057 & 0.0120 & 1.1264 \\
$\boldsymbol{\sigma}$ & 0.0432 & 0.0752 & 0.1098 & 1.0613 \\
$\boldsymbol{C}_{\boldsymbol{v}}$ & 0.0282 & 0.0592 & 0.0684 & 0.8603 \\
$\boldsymbol{S}_{\boldsymbol{k}}$ & 1.0012 & 1.0671 & 1.0071 & 1.0525 \\
$\boldsymbol{K}_{\boldsymbol{s}}$ & 1.0031 & 1.0137 & 1.0163 & 1.0864 \\
\hline
\end{tabular}

Table 2: Quantiles of the NTPLD for some selected parameter values.

\begin{tabular}{|c|c|c|c|}
\hline $\mathrm{U}$ & $(\alpha=3, \theta=2, c=1)$ & $(\alpha=2, \theta=1, c=0.8)$ & $(\alpha=4, \theta=3, c=1)$ \\
\hline 0.1 & 2.329409 & 1.186062 & 3.404192 \\
\hline 0.2 & 2.425832 & 1.274849 & 3.489411 \\
\hline 0.3 & 2.501658 & 1.353477 & 3.552624 \\
\hline 0.4 & 2.570449 & 1.431551 & 3.607519 \\
\hline 0.5 & 2.638186 & 1.514875 & 3.659530 \\
\hline 0.6 & 2.709535 & 1.609860 & 3.712305 \\
\hline 0.7 & 2.790407 & 1.726963 & 3.769819 \\
\hline 0.8 & 2.892102 & 1.889429 & 3.838934 \\
\hline 0.9 & 3.049035 & 2.176375 & 3.939256 \\
\hline
\end{tabular}

\subsection{Mode of the NTPLD}

To get the mode we differentiate the pdf of the NTPLD and equate to zero, so that the mode becomes the root of the equation

$$
x=c\left(\frac{k}{1-k}\right)^{\frac{1}{\alpha}}+\theta
$$

where $k=\frac{\alpha-1}{\alpha^{2}+\alpha}$ 


\subsection{Entropy}

Renyi (1961) defined entropy of a random variable $X$ as the measure of variation of uncertainty. Then given a random variable $X$ with probability density function $f(x)$, the Renyi entropy is defined as:

$$
T_{R}(k)=\frac{1}{1-k} \log \left[\int f^{k}(x) d x\right], k>0, k \neq 1
$$

Then, for the three parameter paralogistic distribution we have that

$$
T_{R}(k)=\frac{1}{1-k} \log \left[\int \frac{\alpha^{2}}{x-\theta}\left(1+\left(\frac{x-\theta}{c}\right)^{\alpha}\right)^{-(\alpha+1)}\left(\frac{x-\theta}{c}\right)^{\alpha}\right]^{k} d x
$$

On further simplification, we have

$$
\begin{gathered}
T_{R}(k)=\frac{1}{1-k} \log \left[\frac{\alpha^{2 k}}{\alpha} \frac{c}{c^{k}} \int \frac{t^{\frac{-k}{\alpha}}}{(1+t)^{(\alpha+1) k}} t^{k} t^{\frac{1-\alpha}{\alpha}} d t\right] \\
=\frac{1}{1-k} \log \left[\alpha^{2 k-1} c^{1-k} \int \frac{t^{\frac{-k}{\alpha}}}{(1+t)^{(\alpha+1) k}} t^{\left(k+\frac{1}{\alpha}-1\right)} d t\right]
\end{gathered}
$$

\section{Maximum likelihood estimation}

The Maximum likelihood estimate (MLE) of a parameter(s) entails maximizing the log -likelihood function of the distribution of the random variable. Let $x_{1}, x_{2}, x_{3}, \ldots, x_{n}$ be a random sample of size $n$ from the NTPLD. Then the log-likelihood function $\ell(x ; \emptyset)$ is given by:

$$
\ell(x ; \emptyset)=\sum_{i=1}^{n} \ln \left[\frac{\alpha^{2}}{x_{i}-\theta}\left(1+\left(\frac{x_{i}-\theta}{c}\right)^{\alpha}\right)^{-(\alpha+1)}\left(\frac{x_{i}-\theta}{c}\right)^{\alpha}\right]
$$

where $\emptyset=(\alpha, \theta, c)$

The score function $\mu(\varnothing)=(\partial \ell / \partial \alpha, \partial \ell / \partial c, \partial \ell / \partial \theta)^{T}$ corresponding to (4.1) are obtained by minimizing equation (4.1) with respect the three parameters respectively.

$$
\begin{aligned}
\frac{\partial \ell(.)}{\partial \alpha} & =\frac{2 n}{\alpha}+\sum_{i=1}^{n} \ln \left(x_{i}-\theta\right)-\ln c-\alpha \sum_{i=1}^{n} \frac{\left.\left(\frac{x_{i}-\theta}{c}\right)^{\alpha} \ln \left(\frac{x_{i}-\theta}{c}\right)\right)}{1+\left(\frac{x_{i}-\theta}{c}\right)^{\alpha}} \\
& +\sum_{i=1}^{n} \ln \left(1+\left(\frac{x_{i}-\theta}{c}\right)^{\alpha}\right)-\frac{\left.\left(\frac{x_{i}-\theta}{c}\right)^{\alpha} \ln \left(\frac{x_{i}-\theta}{c}\right)\right)}{1+\left(\frac{x_{i}-\theta}{c}\right)^{\alpha}}=0
\end{aligned}
$$




$$
\begin{gathered}
\frac{\partial \ell(.)}{\partial \mathrm{c}}=-\frac{n \alpha}{c}+\alpha^{2}\left(x_{i}-\theta\right)^{\alpha} c^{-(\alpha+1)} \sum_{i=1}^{n} \frac{1}{1+\left(\frac{x_{i}-\theta}{c}\right)^{\alpha}} \\
+\alpha(x-\theta)^{\alpha} c^{-(\alpha+1)} \sum_{i=1}^{n} \frac{1}{1+\left(\frac{x_{i}-\theta}{c}\right)^{\alpha}}=0 \\
\frac{\partial \ell(.)}{\partial \theta}=\sum_{i=1}^{n} \frac{1}{x_{i}-\theta}-\alpha \sum_{i=1}^{n} \frac{1}{x_{i}-\theta} \\
+\alpha(\alpha+1)\left(x_{i}-\theta\right)^{\alpha} c^{-(\alpha+1)} \sum_{i=1}^{n} \frac{1}{1+\left(\frac{x_{i}-\theta}{c}\right)^{\alpha}}=0
\end{gathered}
$$

Clearly 4.2, 4.3 and 4.4 is a non-linear system of equations which cannot be solved analytically. However, the system of equations can be solved numerically using iterative procedure such as the Newton-Raphson iterative method. The maximum likelihood estimators $\widehat{\varnothing}$ of $\varnothing$ can be achieved using the Newton Raphson's iterative method which is given by:

$$
\widehat{\emptyset}=\emptyset_{k}-H^{-1}\left(\varnothing_{k}\right)-u\left(\varnothing_{k}\right)
$$

where $H(\varnothing)$ denote the Hessian matrix of the log-likelihood function and $u(\varnothing)$ is the score function. The Hessian matrix is thus defined as:

$$
H(\varnothing)=\frac{\partial u(\emptyset)}{\partial \varnothing \partial \emptyset^{\prime}}=\left(\begin{array}{lll}
\frac{\partial^{2} \ln L}{\partial \alpha^{2}} & \frac{\partial^{2} \ln L}{\partial \alpha \partial c} & \frac{\partial^{2} \ln L}{\partial \alpha \partial \theta} \\
\frac{\partial^{2} \ln L}{\partial c \partial \alpha} & \frac{\partial^{2} \ln L}{\partial c^{2}} & \frac{\partial^{2} \ln L}{\partial c \partial \theta} \\
\frac{\partial^{2} \ln L}{\partial \theta \partial \alpha} & \frac{\partial^{2} \ln L}{\partial \theta \partial c} & \frac{\partial^{2} \ln L}{\partial \theta^{2}}
\end{array}\right)
$$

where $\frac{\partial^{2} \ell(.)}{\partial \alpha^{2}}=\frac{2 n}{\alpha}-\alpha\left(x_{i}-\theta\right) \sum_{i=1}^{n}\left[\frac{\ln c c^{-\alpha}}{\ln \left(\frac{x_{i}-\theta}{c}\right)}+c^{-\alpha} \ln \left(\frac{x_{i}-\theta}{c}\right) \ln \left(x_{i}-\theta\right)\right]$

$$
\begin{gathered}
+\sum_{i=0}^{n}\left(x_{i}-\theta\right)^{\alpha} c^{-\alpha} \ln \left(\frac{x_{i}-\theta}{c}\right)+\sum_{i=1}^{n}\left(\frac{x_{i}-\theta}{c}\right) \ln \left(\frac{x_{i}-\theta}{c}\right) \frac{1}{1+\ln \left(\frac{x_{i}-\theta}{c}\right)^{\alpha}} \\
-\left[\frac{\left(\frac{x_{i}-\theta}{c}\right)^{\alpha} \ln \left(\frac{x_{i}-\theta}{c}\right) \sum_{i=1}^{n}\left(1+\left(\frac{x_{i}-\theta}{c}\right)^{\alpha}\right) \ln \left(\frac{x_{i}-\theta}{c}\right)-\left(\frac{x_{i}-\theta}{c}\right)^{\alpha} \ln \left(\frac{x_{i}-\theta}{c}\right)}{\left(1+\left(\frac{x_{i}-\theta}{c}\right)^{\alpha}\right)^{2}}\right]
\end{gathered}
$$




$$
\begin{aligned}
& \frac{\partial^{2} \ell(.)}{\partial \alpha \partial c}=-\frac{n}{c}-\left(\sum_{i=1}^{n} 1+\left(x_{i}-\theta\right)^{\alpha} \alpha c^{-\alpha}\left(x_{i}-\theta\right)^{\alpha}\left[-c^{-\alpha-1}\right.\right. \\
& \left.-\alpha \ln \left(\frac{x_{i}-\theta}{c}\right) c^{-(\alpha+1)}\right]+\alpha\left(x_{i}-\theta\right)^{\alpha} c^{-\alpha} \ln \left(\frac{x_{i}-\theta}{c}\right)^{\alpha}+\left(x_{i}\right. \\
& \left.-\theta)^{\alpha} c^{-(\alpha+1)}\right) /\left[1+\left(\frac{x_{i}-\theta}{c}\right)^{\alpha}\right]^{2}-\sum_{i=1}^{n} \frac{\alpha\left(x_{i}-\theta\right) c^{-(\alpha+1)}}{1+\left(\frac{\left(x_{i}-\theta\right)}{c}\right)} \\
& +\left(\sum_{i=1}^{n} 1+\left(x_{i}-\theta\right)^{2 \alpha} c^{-\alpha}\left[-c^{-\alpha-1}-\ln \left(\frac{x_{i}-\theta}{c}\right) c^{-(\alpha+1)}\right]\right. \\
& \left.+\left(x_{i}-\theta\right)^{\alpha} c^{-\alpha} \ln \left(\frac{x_{i}-\theta}{c}\right)^{\alpha}\left(x_{i}-\theta\right)^{\alpha} c^{-(\alpha+1)}\right) /\left[1+\left(\frac{x_{i}-\theta}{c}\right)^{\alpha}\right]^{2} \\
& \frac{\partial^{2} \ell(.)}{\partial \alpha \partial \theta}=\frac{2 n}{\alpha}-\sum_{i=1}^{n} \frac{1}{x_{i}-\theta} \\
& +\left(\sum_{i=1}^{n} 1+\left(x_{i}-\theta\right)^{\alpha} c^{-\alpha}\left(-\alpha\left(\frac{x_{i}-\theta}{c}\right)^{\alpha}\right)\left(\frac{1}{x_{i}-\theta}\right)\right. \\
& -\ln \left(\frac{x_{i}-\theta}{c}\right)^{\alpha}\left(x_{i}-\theta\right)^{\alpha} c+\alpha\left(\frac{x_{i}-\theta}{c}\right)^{\alpha} \ln \left(\frac{x_{i}-\theta}{c}\right)^{\alpha}\left(x_{i}\right. \\
& \left.-\theta)^{\alpha-1} c^{-\alpha}\right) /\left[1+\left(\frac{x_{i}-\theta}{c}\right)^{\alpha} c^{-\alpha}\right]^{2} \\
& -\left(\sum_{i=1}^{n} \frac{\alpha\left(x_{i}-\theta\right)^{\alpha-1} c^{-\alpha}}{1+\left(\frac{x_{i}-\theta}{c}\right)^{\alpha}}\right. \\
& +\sum_{i=1}^{n} 1+\left(x_{i}-\theta\right)^{2 \alpha} c^{-\alpha}\left(-\left(\frac{x_{i}-\theta}{c}\right)^{\alpha}\right)\left(\frac{1}{x_{i}-\theta}\right) \ln \left(\frac{x_{i}-\theta}{c}\right)^{\alpha}\left(x_{i}\right. \\
& \left.-\theta)^{\alpha} c+\alpha\left(\frac{x_{i}-\theta}{c}\right)^{\alpha} \ln \left(\frac{x_{i}-\theta}{c}\right)^{\alpha}\left(x_{i}-\theta\right)^{\alpha-1} c^{-\alpha}\right) /[1 \\
& \left.+\left(\frac{x_{i}-\theta}{c}\right)^{\alpha} c^{-\alpha}\right]^{2} \\
& \frac{\partial^{2} \ell(.)}{\partial c \partial \alpha}=-\frac{n}{c}-\left(\sum_{i=1}^{n} 1+\left(x_{i}-\theta\right)^{\alpha} \alpha c^{-\alpha}\left(x_{i}-\theta\right)^{\alpha}\right)\left[-c^{-\alpha-1}\right. \\
& \left.-\alpha \ln \left(\frac{x_{i}-\theta}{c}\right) c^{-(\alpha+1)}\right]
\end{aligned}
$$




$$
\begin{aligned}
& +\alpha\left(x_{i}-\theta\right)^{\alpha} c^{-\alpha} \ln \left(\frac{x_{i}-\theta}{c}\right)^{\alpha}\left(x_{i}-\theta\right)^{\alpha} c^{-(\alpha+1)} /\left[1+\left(\frac{x_{i}-\theta}{c}\right)^{\alpha}\right]^{2} \\
& -\sum_{i=1}^{n} \frac{\alpha\left(x_{i}-\theta\right) c^{-(\alpha+1)}}{1+\left(\frac{x_{i}-\theta}{c}\right)}+\left(\sum_{i=1}^{n} 1+\left(x_{i}-\theta\right)^{2 \alpha} c^{-\alpha}\left[-c^{-\alpha-1}-\ln \left(\frac{x_{i}-\theta}{c}\right) c^{-(\alpha+1)}\right]\right. \\
& +\left(x_{i}-\theta\right)^{\alpha} c^{-\alpha} \ln \left(\frac{x_{i}-\theta}{c}\right)^{\alpha}\left(x_{i}-\theta\right)^{\alpha} c^{-(\alpha+1)} /\left[1+\left(\frac{x_{i}-\theta}{c}\right)^{\alpha}\right]^{2} \\
& \frac{\partial^{2} \ell(.)}{\partial c^{2}}=-\frac{n}{c^{2}}+\alpha^{2}\left(x_{i}-\theta\right)^{\alpha} c^{-(\alpha+1)} \sum_{i=1}^{n} \frac{\alpha\left(x_{i}-\theta\right)^{\alpha} c^{-(\alpha+1)}}{\left[1+\left(\frac{x_{i}-\theta}{c}\right)^{\alpha}\right]^{2}} \\
& -\sum_{i=1}^{n} \frac{\alpha^{2}(\alpha+1)\left(x_{i}-\theta\right) c^{-(\alpha+2)}}{1+\left(x_{i}-\theta\right)^{\alpha} c^{-\alpha}}+\alpha\left(x_{i}-\theta\right) c^{-(\alpha+1)} \sum_{i=1}^{n} \frac{\alpha\left(x_{i}-\theta\right)^{\alpha} c^{-(\alpha+1)}}{\left[1+\left(\frac{x_{i}-\theta}{c}\right)^{\alpha}\right]^{2}} \\
& -\sum_{i=1}^{n} \frac{\alpha(\alpha+1)\left(x_{i}-\theta\right)^{\alpha} c^{-(\alpha+2)}}{1+\left(\frac{x_{i}-\theta}{c}\right)^{\alpha}} \\
& \frac{\partial^{2} \ell(.)}{\partial c \partial \theta}=-\sum_{i=1}^{n} \frac{2 \alpha(\alpha+1)\left(x_{i}-\theta\right)^{\alpha} c^{-(\alpha+2)}}{1+\left(\frac{x_{i}-\theta}{c}\right)^{\alpha}}+\alpha(\alpha+1)\left(x_{i}-\theta\right)^{\alpha} c^{-(\alpha+1)} \\
& \times \sum_{i=1}^{n} \frac{\alpha\left(x_{i}-\theta\right)^{\alpha} c^{-(\alpha+1)}}{\left[1+\left(\frac{x_{i}-\theta}{c}\right)^{\alpha}\right]^{2}} \\
& \frac{\partial^{2} \ell(.)}{\partial \theta \partial \alpha}=\frac{2 n}{\alpha}-\sum_{i=1}^{n} \frac{1}{x_{i}-\theta}+\left(\sum_{i=1}^{n} 1+\left(x_{i}-\theta\right)^{\alpha} c^{-\alpha}\left(-\alpha\left(\frac{x_{i}-\theta}{c}\right)^{\alpha}\right)\left(\frac{1}{x_{i}-\theta}\right)\right. \\
& -\ln \left(\frac{x_{i}-\theta}{c}\right)^{\alpha}\left(x_{i}-\theta\right)^{\alpha} c+\alpha\left(\frac{x_{i}-\theta}{c}\right)^{\alpha} \ln \left(\frac{x_{i}-\theta}{c}\right)^{\alpha}\left(x_{i}-\theta\right)^{\alpha-1} c^{-\alpha} /[1 \\
& \left.+\left(\frac{x_{i}-\theta}{c}\right)^{\alpha} c^{-\alpha}\right]^{2} \\
& -\left(\sum_{i=1}^{n} \frac{\alpha\left(x_{i}-\theta\right)^{\alpha-1} c^{-\alpha}}{1+\left(\frac{x_{i}-\theta}{c}\right)^{\alpha}}\right. \\
& \left.+\sum_{i=1}^{n} 1+\left(x_{i}-\theta\right)^{2 \alpha} c^{-\alpha}\right)\left(-\left(\frac{x_{i}-\theta}{c}\right)^{\alpha}\right)\left(\frac{1}{x_{i}-\theta}\right) \ln \left(\frac{x_{i}-\theta}{c}\right)^{\alpha}\left(x_{i}\right. \\
& \left.-\theta)^{\alpha} c+\alpha\left(\frac{x_{i}-\theta}{c}\right)^{\alpha} \ln \left(\frac{x_{i}-\theta}{c}\right)^{\alpha}\left(x_{i}-\theta\right)^{\alpha-1} c^{-\alpha}\right) /[1 \\
& \left.+\left(\frac{x_{i}-\theta}{c}\right)^{\alpha}\right]^{2}
\end{aligned}
$$




$$
\begin{aligned}
\frac{\partial^{2} \ell(.)}{\partial \theta \partial c}=-\sum_{i=1}^{n} & \frac{2 \alpha(\alpha+1)\left(x_{i}-\theta\right)^{\alpha} c^{-(\alpha+2)}}{1+\left(\frac{x_{i}-\theta}{c}\right)^{\alpha}}+\alpha(\alpha+1)\left(x_{i}-\theta\right)^{\alpha} c^{-(\alpha+1)} \\
& \times \sum_{i=1}^{n} \frac{\alpha\left(x_{i}-\theta\right)^{\alpha} c^{-(\alpha+1)}}{\left(1+\left(\frac{x_{i}-\theta}{c}\right)^{\alpha}\right)^{2}} \\
\frac{\partial^{2} \ell(.)}{\partial \theta^{2}}=\sum_{i=1}^{n} \frac{1}{\left(x_{i}-\theta\right)^{2}}+\sum_{i=1}^{n} \frac{\alpha}{\left(x_{i}-\theta\right)^{2}}+\alpha(\alpha+1)\left(x_{i}-\theta\right)^{\alpha} c^{-(\alpha+1)} & \times \sum_{i=1}^{n} \frac{\alpha c^{-\alpha}\left(x_{i}-\theta\right)^{\alpha-1}}{\left[1+\left(\frac{x_{i}-\theta}{c}\right)^{\alpha}\right]^{2}}-\sum_{i=1}^{n} \frac{\alpha^{2}(\alpha+1) c^{-(\alpha+1)}\left(x_{i}-\theta\right)^{\alpha-1}}{1+\left(\frac{x_{i}-\theta}{c}\right)^{\alpha}}
\end{aligned}
$$

\section{Simulation study on the mle}

In this section, we investigate the performance and accuracy of the MLE of the NTPLD. This was achieved by simulation studies of the model for different sample sizes. Random data from the NTPLD are generated using equation (3.10). We conducted 1000 Monte Carlo simulation for each sample sizes $\mathrm{n}=25,50,100,200$ and parameter values $(\alpha=3, \theta=2, c=1),(\alpha=2, \theta=1, c=0.8),(\alpha=4, \theta=3, c=1)$. An algorithm for the simulation study is given by the following steps.

1. Choose the value M (i.e. Number of Monte-Carlo simulation);

2. Choose the values $\emptyset_{0}=\left(\alpha_{0}, \theta_{0}, c_{0}\right)$ corresponding to the parameters of the NTPLD $(\alpha, \theta, c)$.

3. Generate a sample of size $n$ from the NTPLD

4. Compute the maximum likelihood estimates $\widehat{\emptyset}$ of $\varnothing$;

5. Repeat steps (3-4) M times.

6. Compute the bias and the mean square error (MSE)

where bias $=E(\widehat{\varnothing})-\emptyset$ and $\mathrm{MSE}=E(\widehat{\varnothing}-\emptyset)^{2}$ 
Table 3: Summary Statistics of the bias and MSE for some selected parameter values $(\alpha, \theta, c)$.

\begin{tabular}{cccccccc}
\hline Parameters & $\mathrm{n}$ & $\operatorname{Bias}(\alpha)$ & $\operatorname{MSE}(\alpha)$ & $\operatorname{Bias}(\theta)$ & $\operatorname{MSE}(\theta)$ & $\operatorname{Bias}(c)$ & $\operatorname{MSE}(c)$ \\
\hline$\alpha=3$ & 25 & 0.0000701 & 0.6114014 & 0.0094425 & 0.0214631 & -0.0005231 & 0.0496241 \\
$\theta=2$ & 50 & 0.0000113 & 0.2737522 & 0.0040610 & 0.0205418 & -0.0004640 & 0.0230552 \\
$\mathrm{C}=1$ & 100 & -0.000093 & 0.1700612 & -0.000332 & 0.0181146 & -0.0003712 & 0.0208761 \\
& 200 & -0.000014 & 0.0865626 & -0.000134 & 0.0024914 & -0.0000183 & 0.0066515 \\
& & & & & & & \\
$\alpha=2$ & 25 & 0.0000481 & 0.4478364 & 0.0302780 & 0.0145053 & -0.0004301 & 0.0631763 \\
$\theta=4$ & 50 & -0.000250 & 0.0879272 & 0.0207105 & 0.0034381 & -0.0002390 & 0.0218192 \\
$\mathrm{C}=0.8$ & 100 & -0.000113 & 0.0323231 & 0.0082718 & 0.0009805 & -0.0001061 & 0.0075915 \\
& 200 & -0.000136 & 0.0139825 & 0.0080890 & 0.0004832 & -0.0001058 & 0.0040412 \\
& & & & & & & \\
$\alpha=4$ & 25 & 0.0474912 & 1.3470941 & 0.0474915 & 0.0306123 & -0.0052119 & 0.1990663 \\
$\theta=3$ & 50 & 0.0255746 & 0.9993062 & -0.0081890 & 0.0244457 & -0.0031942 & 0.0465727 \\
$\mathrm{C}=1$ & 100 & -0.0004340 & 0.5261974 & -0.0073881 & 0.0116922 & -0.0005172 & 0.0218631 \\
& 200 & -0.0004313 & 0.3530275 & -0.0052644 & 0.0095113 & -0.0000608 & 0.0189441 \\
\hline
\end{tabular}

From Table 3, we clearly observe that the values of the bias and the MSE of the parameter estimates decreases as the sample size $\mathrm{n}$ increases.

\section{Application to real data}

Here we compare the fit of the NTPLD to some other related distributions such as: the paralogistic, gamma, transformed beta, Log-logistic and inverse paralogistic distribution in fitting the breaking stress of carbon fibers (in Gab) reported in Nicholas and Padgett (2006). The data is given in Table 4 .

Table 4. The breaking stress of carbon fibres (in Gab)

\begin{tabular}{llllllllll}
\hline 3.70 & 2.74 & 2.73 & 2.50 & 3.60 & 3.11 & 3.27 & 2.87 & 1.47 & 3.11 \\
3.56 & 4.42 & 2.41 & 3.19 & 3.22 & 1.69 & 3.28 & 3.09 & 1.87 & 3.15 \\
4.90 & 1.57 & 2.67 & 2.93 & 3.22 & 3.39 & 2.81 & 4.20 & 3.33 & 2.55 \\
3.31 & 3.31 & 2.85 & 1.25 & 4.38 & 1.84 & 0.39 & 3.68 & 2.48 & 0.85 \\
1.61 & 2.79 & 4.70 & 2.03 & 1.89 & 2.88 & 2.82 & 2.05 & 3.65 & 3.75 \\
2.43 & 2.95 & 2.97 & 3.39 & 2.96 & 2.35 & 2.55 & 2.59 & 2.03 & 1.61 \\
2.12 & 3.15 & 1.08 & 2.56 & 1.80 & 2.53 & & & & \\
\hline
\end{tabular}

Source: Nicholas and Padgett (2006). 
The MLE of the NTPLD of the data along-side the standard error of estimates of the parameters of each distribution and some goodness-of-fit statistics are reported in Table 5.

Table 5: Summary Statistics of the distributions for the data set

\begin{tabular}{|c|c|c|c|c|}
\hline Distribution & $\begin{array}{l}\text { Estimated } \\
\text { Parameter }\end{array}$ & $2 \log L$ & $A I C$ & $K-S$ \\
\hline \multirow{3}{*}{ NTPLD } & $\alpha=5.6679$ & & & \\
\hline & $c=5.7879$ & 170.6155 & 176.6155 & 0.0701 \\
\hline & $\theta=1.2608$ & & & \\
\hline \multirow[t]{2}{*}{ Paralogistic } & $\alpha=3.9690$ & 173.5674 & 177.5674 & 0.0799 \\
\hline & $c=4.1531$ & & & \\
\hline \multirow[t]{3}{*}{ Gamma } & $\alpha=7.4873$ & 182.3351 & 186.3351 & 0.1328 \\
\hline & $c=2.7131$ & & & \\
\hline & $\alpha=7.4873$ & & & \\
\hline \multirow[t]{3}{*}{ Trbeta } & $\beta=4.8920$ & 171.6909 & 179.3818 & 0.0773 \\
\hline & $\lambda=0.6248$ & & & \\
\hline & $c=5.1506$ & & & \\
\hline \multirow[t]{2}{*}{ Llogis } & $\alpha=7.4873$ & 183.2905 & 187.2905 & 0.0939 \\
\hline & $c=5.1506$ & & & \\
\hline \multirow[t]{2}{*}{ Invparalogis } & $\alpha=2.8586$ & 205.2754 & 209.2754 & 0.1683 \\
\hline & $c=1.6923$ & & & \\
\hline
\end{tabular}

The density plot representing the density function of the fitted distribution, the plot of the CDF of the empirical and fitted distribution, the Q-Q plot representing the empirical quantiles against the theoretical quantiles and the P-P Plot representing the empirical distribution function evaluated at each data plot against the fitted distribution function for the NTPLD, paralogistic, gamma, transformed beta, Log-logistic and inverse paralogistic distributions are given in the Figures 4, 5 and 6 respectively. 

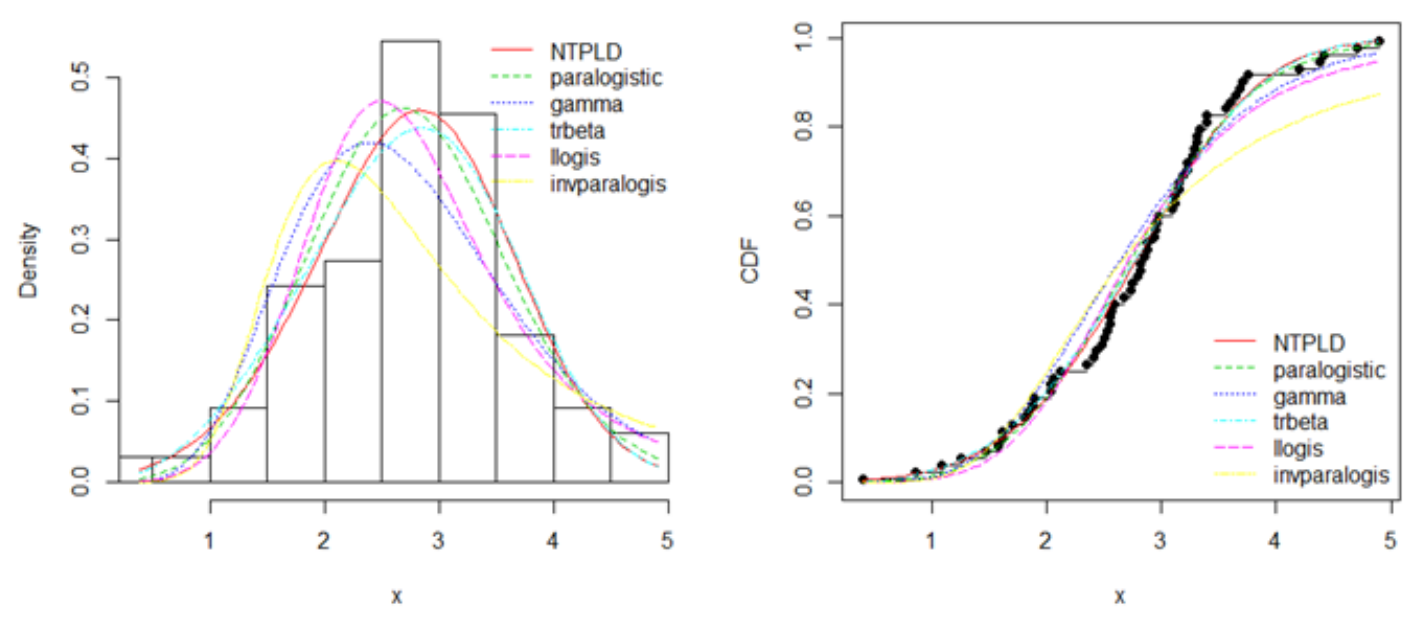

Figure 4: The density plot and CDF of the fitted distribution for the data set
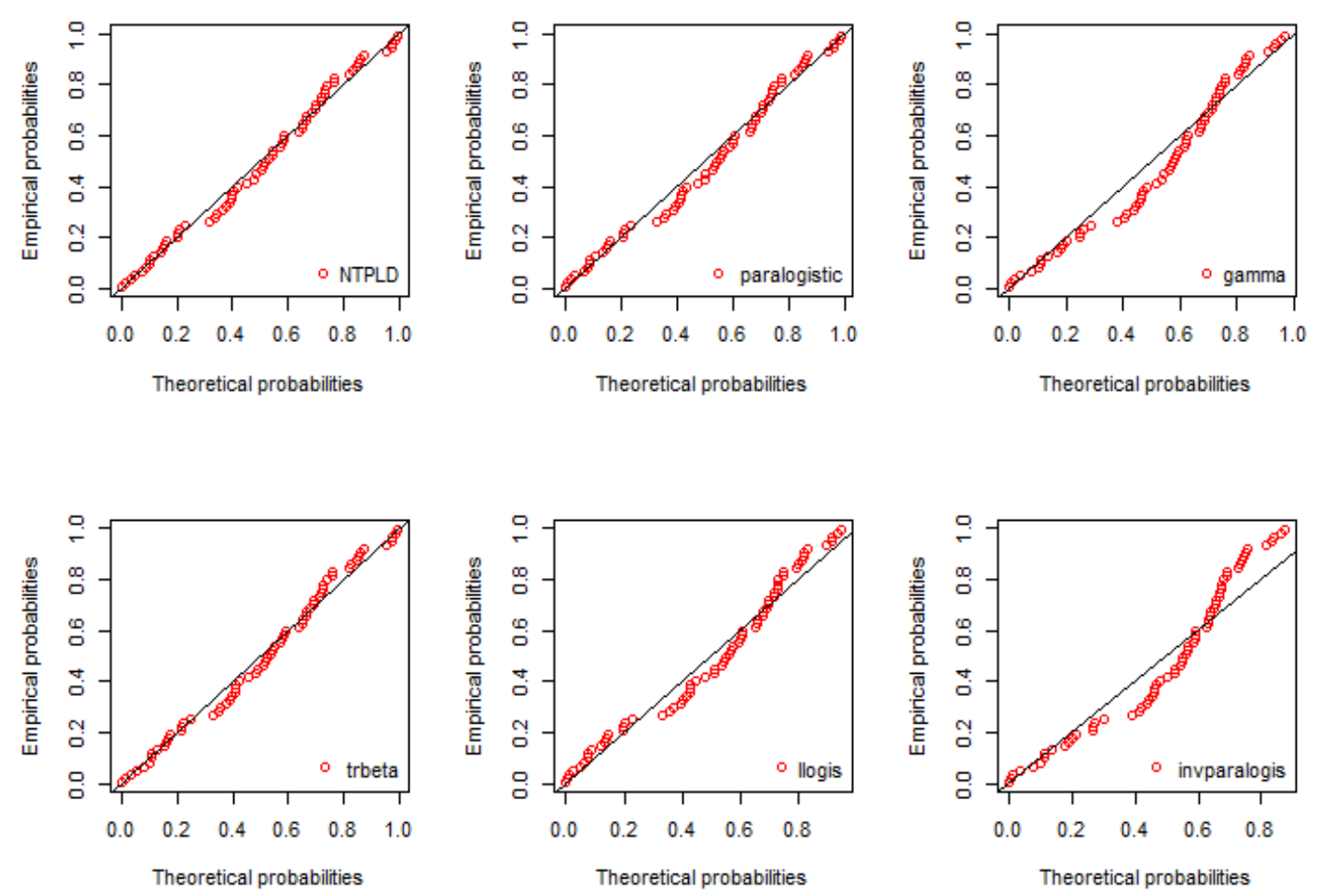

Figure 5: The P-P plot of the distributions for the data set. 


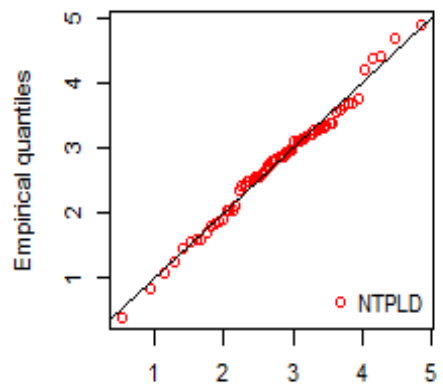

Theoretical quantiles

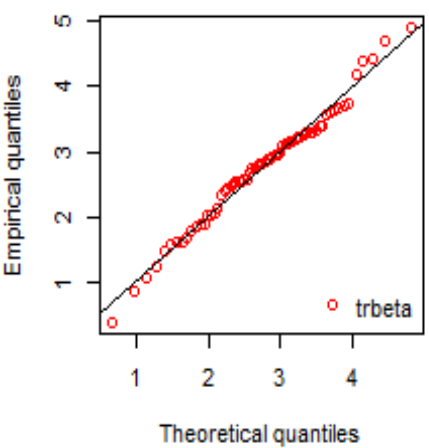

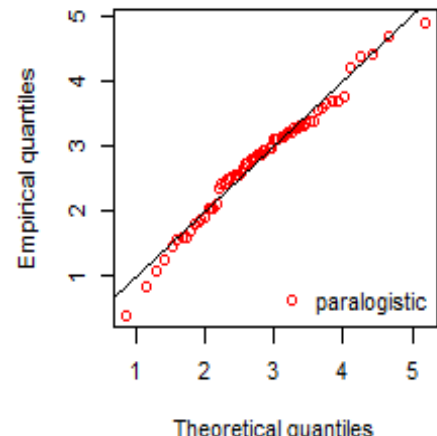

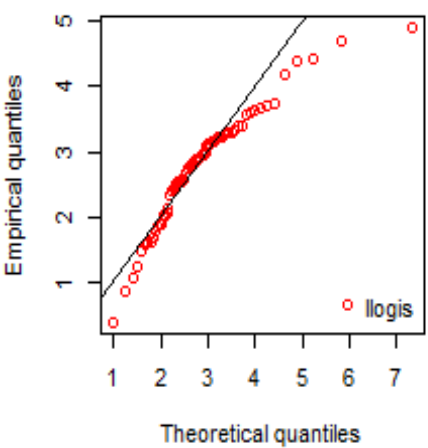

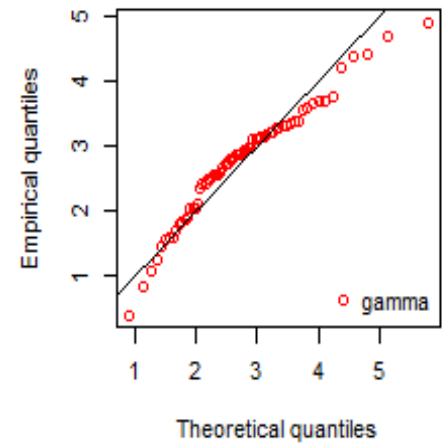

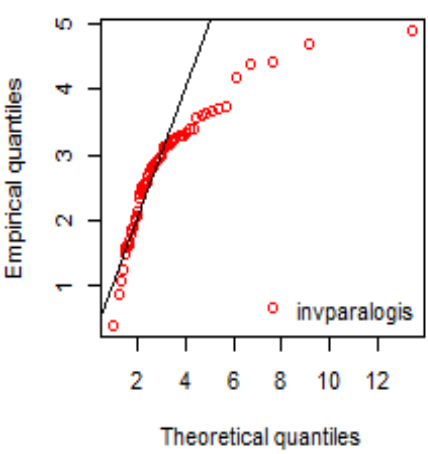

Figure 6: The Q-Q plots of the distributions for the data set

\section{Discussion of results}

The results obtained in Table 5 show that the NTPLD has the smaller AIC value as well the K-S statistic value which supports that the NTPLD provides a good fit for the data. Similarly, in Figures 4, 5 and 6, it is clearly evident that the performance of the NTPLD is better than the Paralogistic, gamma, transformed beta, Log-logistic and inverse paralogistic distributions in terms of the density plots, the P-P plots and the Q-Q plots respectively.

\section{Conclusion}

We proposed a NTPLD for fitting lifetime data. MLE of the model parameters performed accurately. This newly proposed distribution appears to be superior to the Paralogistic, gamma, transformed beta, Log-logistic and inverse paralogistic distributions as demonstrated by the density plots, the P-P plots and the Q-Q plots in fitting a lifetime data that was considered in this paper. 


\section{References}

[1] Alshawarbeh, E., Lee, C., and Famoye, F. (2012). The Beta-Cauchy distribution. Journal of Probability and Statistical Science, 10, 41-58.

[2] Guerra, R. R., Pena-Ramirez, F. A. and Cordeiro, G. M. (2017). The gamma Burr XII distributions: Theory and applications, Journal of Data Science, 15, 467-494.

[3] Hamed, D., Famoye, F. and Lee, C. (2018). On Families of Generalized Pareto Distributions: Properties and Applications, Journal of Data Science, 16(2), 377-396.

[4] Ismail, N. H. B. and Khalid, Z. B. M. (2014). EM algorithm in estimating the 2- and 3-parameter Burr type III distribution. AIP Conference proceeding, 1605, 881-887.

[5] Kleiber, C. and Kotz, S. (2003). Statistical Size Distributions in Economics and Actuarial Sciences. John Wiley and Sons, New York.

[6] Klugman, S. and Persa. R. (1999). Fitting bivariate loss distributions with copulas, Insurance Mathematics and Economics, 24, 139-148.

[7] Mahomoudi, E. (2011). The beta generalized pareto distribution with application to lifetime data. Mathematics and computers in simulation, 81, 2014-2430.

[8] Maksaei, N. and Aitun, E. (2017). The Generalized Weibull-Burr XII Distribution and its application. Journal of Data Science, 16, 535-552.

[9] Makubate, B. N. D., Oluyede, B. O. and Fagbemigbe, F. A. (2018). The Exponentiated Burr XII Weibull Disribution: Model, Properties and Applications, Journal of Data Science, 16(3), 431 $-462$.

[10] Mc-Donald, J. B. (1984). Some generalized functions for the Size distributions in Economics and Actuarial Sciences, John Wiley and sons, New York.

[11] Nadarajah, S. and Kotz, S. (2006). The beta exponential distribution. Reliability and Engineering System and Safety, 91, 689-697.

[12] Nicholas, M. D. and Padgett, W. J. (2006). A bootstrap control chart for Weibull percentiles Quality and Reliability engineering international, 22, 141-151. 
[13] Renyi, A. (1961). On measurement of entropy and information, proceeding of the 4th Berkeley Symposium on mathematical statistics and probability university of California press, Berkeley, 1 , 547-561.

[14] Silva, G. O., Ortega, E. M. M. and Cordeiro, G. M. (2010). The beta modified Weilbull distribution. Lifetime Data Analysis, 16, 409-430. 
